\title{
Indication and Timing of Surgery Are Essential in Stabilization of the Chest Wall in Flail Chest Trauma Patients
}

\author{
Ali Imad El-Akkawi1, Frank Vincenzo de Paoli'1,2, Morten Bendixen', \\ Thomas Decker Christensen ${ }^{*}$
}

\author{
${ }^{1}$ Department of Cardiothoracic and Vascular Surgery, Department of Clinical Medicine, Aarhus University Hospital, Aarhus, \\ Denmark \\ ${ }^{2}$ Department of Biomedicine, Aarhus University, Aarhus, Denmark \\ Email: *tdc@din.au.dk
}

How to cite this paper: El-Akkawi, A.I., de Paoli, F.V., Bendixen, M. and Christensen, T.D. (2018) Indication and Timing of Surgery Are Essential in Stabilization of the Chest Wall in Flail Chest Trauma Patients. Open Journal of Thoracic Surgery, 8, 79-85. https://doi.org/10.4236/ojts.2018.84014

Received: September 27, 2018

Accepted: November 30, 2018

Published: December 3, 2018

Copyright $\odot 2018$ by authors and Scientific Research Publishing Inc. This work is licensed under the Creative Commons Attribution International License (CC BY 4.0)

http://creativecommons.org/licenses/by/4.0/

\begin{abstract}
Flail chest occur after blunt trauma to the thorax. Most often treatment of flail chest is conservative with analgesia and respiratory support, if needed. New plate systems and surgical approaches have improved outcomes after surgery. Surgical treatment of flail chest is associated with a reduced risk of severe pneumonia, shorter time with mechanical ventilation and a reduced length of stay in the Intensive Care Unit (ICU) compared to conservative treatment. However, currently approximately $1 \%$ of patients with flail chest undergo surgery. We are presenting two cases of flail chest treated surgically by fixating the most dislocated posterior fractures. One patient avoided mechanical ventilation, and the other patient was quickly weaned from respirator after surgery. We found that surgical stabilization of posterior fractures in patients with flail chest is a safe method with a high possibility of positive outcomes for the patients. Surgical stabilization of flail chest is indicated in patients with consistent pain (case 1) and increased risk of pneumonia, respiratory failure or prolonged mechanical ventilation (case 2). Furthermore, it was possible to achieve stable thorax wall by only fixating the most dislocated posterior fractures in the flail segment.
\end{abstract}

\section{Keywords}

Flail-Chest, Surgery, Ribfix, Costa, Fracture

\section{Introduction}

Clinically, flail chest is defined as paradox movement of a segment of the tho- 
racic wall during spontaneous breathing, and radio graphically with fractures of three or more consecutive ribs or costal cartilages in two or more places [1].

Most often treatment of flail chest is conservative, with primarily analgesia, e.g. epidural pain catheter (EDC), and respiratory support if needed [2]. Some patients may need mechanical ventilation and admission to an Intensive Care Unit (ICU), depending primarily on the severity of the trauma [2]. Prolonged stay in the ICU and mechanical ventilation is associated with an increased risk of complications such as respiratory distress and severe pneumonia [3].

Although increased recognition and utilization of surgical treatment is known in literature, less than $1 \%$ of patients with flail chest under gosurgery [4]. The reasons for this incongruity are myriad, e.g. lack of evidence-based indications for surgery, lack of familiarity with surgical techniques and lack of specialty ownership for the care of rib fracture patients [5].

Several surgical techniques and plate systems have been used to stabilize flail chest over the years [5]. Accordingly, Nirula et al. (2009) described six commonly used surgical approaches [6]. Despite the risk of bleeding and infection, most surgical approaches have shown superiority compared to conservative treatment in regard to hospital stay, time with mechanical ventilation and risk of pneumonia. However, there are no data supporting one technique over another [7]. Thus, multiple fixations of cost a fractures can theoretically result in a rigid thorax and consequently respiratory restrictions and pain [8]. In contrast, it has been suggested that fixation of a single fracture reduces the flail segment to a simple cost a fracture [8]. Accordingly, in the presented cases we only applied plates on the most severely dislocated posterior fractures in order to achieve stabilization of a flail segment without inhibiting movement of the thoracic wall.

The purpose of this case report is to present the positive outcomes of surgical stabilization of flail chest by only fixating the posterior fractures in a flail segment.

\section{Case 1}

A 41 years old man was stepped on by a horse on the right hemi thorax and subsequently admitted to the local hospital. The patient was healthy and a non-smoker, but had a consumption of 20 units of alcohol on a weekly basis. At admission, the patient was hemodynamic and respiratory stable. The patient had a clinical flail chest with paradox movement of the right hemi thorax, and additionally, pneumothorax on the right side, subcutaneous emphysema and lack of breathing sounds on the right hemi thorax at auscultation. Vital parameters were inconspicuous, except from a respiration frequency per minute of 40 . The pneumothorax was primarily treated with a chest tube (size 24 French) to stabilize the breathing prior to a computed tomography (CT) scan. The trauma CT revealed single and double cost a fractures of costa 8 - 11 and $4-7$, respectively, on the right hemi thorax. After the CT scan the patient was treated with an EDC and averted mechanical ventilation. The next day, the patient was moved to 
Aarhus University Hospital, Denmark.

The preoperative CT scan is displayed in Figure 1.

At day 4 the chest tube was removed and at day 9 the EDC was turned off. However, due to continued paradox thoracic movement and insufficient pain management it was decided to surgically stabilize the right hemi thorax by osteosynthesis.

The CT scan one year after surgery is shown in Figure 2. The posterior dislocated fractures of costa 6 - 8 were fixated with RibFixBlu ${ }^{\mathrm{TM}}$ (Thoracic Fixation System by Zimmer Biomet, Indiana USA). The procedure was performed through a posterolateral muscle-sparring incision. The Latissimus dorsi muscle was retracted posteriorly and the serratus anterior muscle was divided in parallel with muscle fiber direction. Periosteal dissection was performed to expose the fractured ribs. A template was used intra operatively to bend the plates in accordance to cost a curvature followed by reposition of the fractures. The RibFix$\mathrm{Blu}^{\mathrm{TM}}$ plates were placed and fixated after removing all interposition tissue. Postoperatively, the thorax was stable and without paradox movement and the patient was discharged 3 days after surgery. At 1 year follow up in the outpatient clinic the patient still needed mild oral analgesics, but he had experienced a significant improvement regarding pain level and was able to maintain his normal job.

\section{Case 2}

A healthy 47 years old man was hit by a truck on the highway. He was otherwise healthy and no history of smoking, but with a moderate consumption of alcohol. The patient was acutely admitted to the emergency room (ER) after pre-hospital intubation. At arrival, the airways were free of obstruction and breathing was stable. However, the patient had circulatory distress, with a low blood pressure of 60/30. A trauma CT revealed a left sided hemothorax and costae fractures on 1 - 12 and 1 - 10 on the left and right side, respectively. Additional injuries were: left scapula fracture, bilateral clavicular fractures, bilateral orbital fractures, several fractures of the cervical spine, a non-dislocated cranial fracture on the right side and subdural hematoma. Furthermore, the patient had a sub capsular hematoma surrounding the left kidney and spleen. A 3D reconstruction of the trauma CT scan is displayed in Figure 3.

In order to stabilize the patient an emergency thoracotomy and laparotomy was performed at the ER. Hemostasis was achieved and the patient was admitted at the ICU for further observation before continuing with surgery on the cervical spine. After 16 daysat the ICU with mechanical ventilation the patient was stable, however, weaning the patient from mechanical ventilation was not possibile. Therefore, it was decided to surgically stabilize the cost a fractures. The surgery was performed through a left sided posterolateral muscle sparing incision to expose the posterior fractures of costa 5 - 7. By using a template intraoperatively, the RibFixBlu ${ }^{\mathrm{TM}}$ was shaped according to the curvature of the costa. Postoperative 


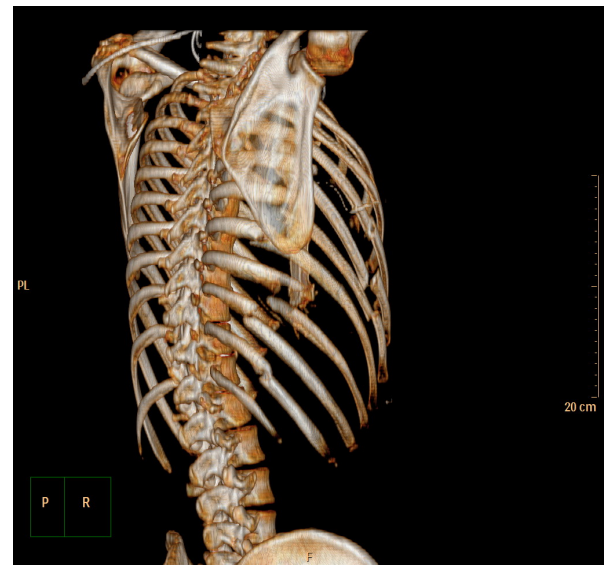

Figure 1. 3D reconstruction of the trauma CT scan showing multiple costa fractures.

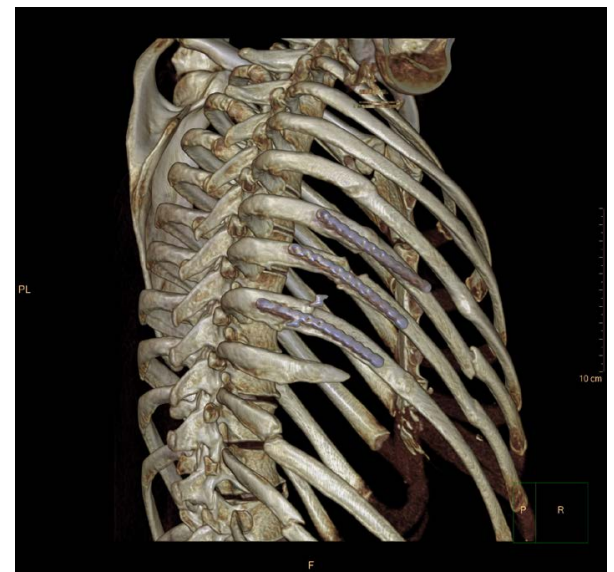

Figure 2. Postoperative 3D reconstruction of CT scan at 1 year follow up.

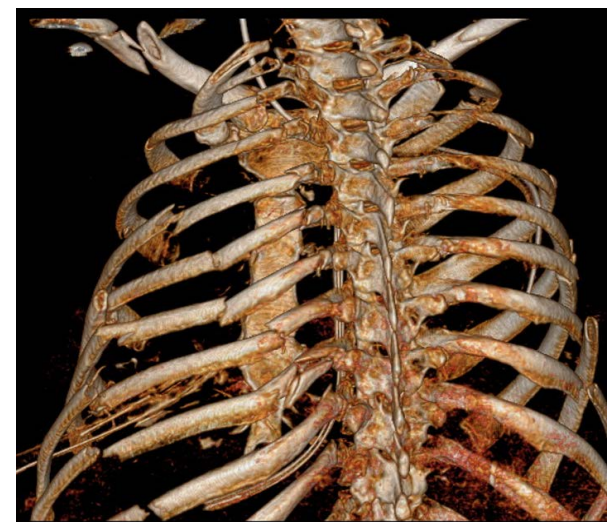

Figure 3. 3D reconstruction of the trauma CT scan showing multiple costa fractures.

tu the patient was weaned from mechanical ventilation and was referred for rehabilitation. Six months after the surgery the patient was seen at our out-patient clinic without pain or respiratory symptoms and had returned to work. 


\section{Discussion}

Several surgical and non-surgical strategies have been applied in the treatment of flail chest over the years [5]. Early surgical techniques have not shown superiority compared to conservative treatment and mechanical ventilation.

However, new plate systems have improved surgical outcomes in the treatment of flail chest [9]. Previous small randomized, controlled trials have shown potential positive outcomes after surgical treatment of flail chest, such as shorter time with mechanical ventilation, shorter time in the ICU and lower rates of severe pneumonia [10] [11]. Tanaka et al. published a randomized controlled trial [11] revealing a significantly higher percentage of surgical patients (61\%) returning to full time employment after 6 months compared to the conservative group (5\%). A recent meta-analysis of three randomized, controlled trials showed a significant reduction of pneumonia, shorter hospital stay, reduction of time with mechanical ventilation and time at ICU [7]. Furthermore, a recent consensus statement recommends surgical stabilization of fractures in the treatment of flail chest [12]. In order to avoid callus and inflammation by the dislocated fractures, it is recommended to surgically stabilize flail chest within 72 hours [12]. The latter statement is, however, based on low level of evidence due to a low number of cases and studies.

In both cases of this report the patients was not operated within 72 hours. At first it was decided to treat the patient of case 1 conservatively. But due to continued paradox thoracic wall movement and inconsistent pain management it was decided to operate the patient 9 days after the trauma. In future cases of traumatic flail chest, it must be considered to undergo surgery earlier in order to reduce admission time and rehabilitation time for the patient. However, selecting the patients for surgery can be difficult depending on the position of the fractures and the severity of the trauma. In case 2 , we were not able to operate the patient before 16 days after the trauma. This was due to, other injuries needing treatment prior to surgery on the thoracic wall.

In concord with Coughlin et al. (2016) surgical stabilization of flail chest in these two cases ensuredfast weaning from mechanical ventilation, respectively. Surgical stabilization of flail chestis indicated in patients with consistent pain (case 1) and increased risk of pneumonia, respiratory failure or prolonged mechanical ventilation (case 2). As previously recommended early surgical stabilization is important, however other life-threatening injuries should be treated prior to surgery on the chest wall (case 2). Furthermore, it was possible to achieve sufficient stability of the thoracic wall by only fixating the most dislocated posterior fractures in the flail segment as previously suggested [8].

\section{Conclusion}

Surgical stabilization of posterior fractures in patients with flail chest is a safe approach with a high possibility of positive outcomes for the patients. However, 
there is a need for larger randomized, and controlled trials to clarify the benefits of surgical treatment of flail chest. Indeed, there is a need of studies comparing different surgical approaches although such studies will be difficult to conduct due to a low number of surgical cases.

\section{Conflicts of Interest}

Thomas Decker Christensen has been on the speaker bureaus for Astra Zeneca, Boehringer-Ingelheim, Pfizer, Roche Diagnostics, Takeda and Bristol-Myers Squibb and has been in an Advisory Board for Bayer and Merck Sharp \& Dohme (MSD).

\section{References}

[1] Ciraulo, D.L., Elliott, D., Mitchell, K.A. and Rodriguez, A. (1994) Flail Chest as a Marker for Significant Injuries. Journal of the American College of Surgeons, 178, 466-470

[2] Simon, B., Ebert, J., Bokhari, F., et al. (2012) Management of Pulmonary Contusion and Flail Chest: An Eastern Association for the Surgery of Trauma Practice Management Guideline. The Journal of Trauma and Acute Care Surgery, 73, S351-S361. https://doi.org/10.1097/TA.0b013e31827019fd

[3] Park, H.O., Kang, D.H., Moon, S.H., Yang, J.H., Kim, S.H. and Byun, J.H. (2014) Risk Factors for Pneumonia in Ventilated Trauma Patients with Multiple Rib Fractures. The Korean Journal of Thoracic and Cardiovascular Surgery, 50, 346-354. https://doi.org/10.5090/kjtcs.2017.50.5.346

[4] Dehghan, N., de Mestral, C., McKee, M.D., Schemitsch, E.H. and Nathens, A. (2014) Flail Chest Injuries: A Review of Outcomes and Treatment Practices from the National Trauma Data Bank. The Journal of Trauma and Acute Care Surgery, 76, 462-468. https://doi.org/10.1097/TA.0000000000000086

[5] Bemelman, M., Poeze, M., Blokhuis, T.J. and Leenen, L.P. (2010) Historic Overview of Treatment Techniques for Rib Fractures and Flail Chest. European Journal of Trauma and Emergency Surgery. Official Publication of the European Trauma Society, 36,407-415. https://doi.org/10.1007/s00068-010-0046-5

[6] Nirula, R., Diaz Jr., J.J., Trunkey, D.D. and Mayberry, J.C. (2009) Rib Fracture Repair: Indications, Technical Issues, and Future Directions. World Journal of Surgery, 33, 14-22. https://doi.org/10.1007/s00268-008-9770-y

[7] Coughlin, T.A., Ng, J.W., Rollins, K.E., Forward, D.P. and Ollivere, B.J. (2016) Management of Rib Fractures in Traumatic Flail Chest: A Meta-Analysis of Randomised Controlled Trials. The Bone \& Joint Journal, 98, 1119-1125. https://doi.org/10.1302/0301-620X.98B8.37282

[8] Marasco, S.F., Davies, A.R., Cooper, J., et al. (2013) Prospective Randomized Controlled Trial of Operative Rib Fixation in Traumatic Flail Chest. Journal of the American College of Surgeons, 216, 924-932. https://doi.org/10.1016/j.jamcollsurg.2012.12.024

[9] Wijffels, M.M.E., Hagenaars, T., Latifi, D., Van Lieshout, E.M.M. and Verhofstad, M.H.J. (2018) Early Results after Operatively versus Non-Operatively Treated Flail Chest: A Retrospective Study Focusing on Outcome and Complications. European Journal of Trauma and Emergency Surgery, 1-9. https://doi.org/10.1007/s00068-018-0961-4 
[10] Granetzny, A., Abd El-Aal, M., Emam, E., Shalaby, A. and Boseila, A. (2005) Surgical versus Conservative Treatment of Flail Chest. Evaluation of the Pulmonary Status. Interactive Cardiovascular and Thoracic Surgery, 4, 583-587. https://doi.org/10.1510/icvts.2005.111807

[11] Tanaka, H., Yukioka, T., Yamaguti, Y., et al.(2002) Surgical Stabilization of Internal Pneumatic Stabilization? A Prospective Randomized Study of Management of Severe Flail Chest Patients. The Journal of Trauma, 52, 727-732.

https://doi.org/10.1097/00005373-200204000-00020

[12] Pieracci, F.M., Majercik, S., Ali-Osman, F., et al. (2017) Consensus Statement: Surgical Stabilization of Rib Fractures Rib Fracture Colloquium Clinical Practice Guidelines. Injury, 48, 307-321. https://doi.org/10.1016/j.injury.2016.11.026 